\title{
A new method to determine the energy scale for high-energy cosmic rays using radio measurements at the Pierre Auger Observatory
}

\author{
Raphael Krause $^{* a}$ for the Pierre Auger Collaboration ${ }^{b}$ \\ ${ }^{a}$ III. Physikalisches Institut A, RWTH Aachen University, Aachen, Germany \\ ${ }^{b}$ Observatorio Pierre Auger, Av. San Martín Norte 304, 5613 Malargüe, Argentina \\ E-mail: auger_spokespersons@fnal.gov \\ Full author list: http://www.auger.org/archive/authors_icrc_2017.html
}

\begin{abstract}
Coherent radio signals in the $\mathrm{MHz}$ range are emitted from extensive air showers initiated by highenergy cosmic rays. Observing this emission enables precise measurement of the energy of the primary particle. Compared with those made with the well-established fluorescence technique, radio measurements are less dependent on atmospheric conditions, and thus offer the potential of energy determination with reduced systematic uncertainties. Combining these accurate measurements with absolute predictions of the radio signal by first-principle calculations based on classical electrodynamics then allows a determination of the cosmic-ray energy scale.

This approach is discussed in the context of the Auger Engineering Radio Array (AERA), sited at the Pierre Auger Observatory. More than 150 autonomous radio stations, covering about $17 \mathrm{~km}^{2}$, are used to record radio emission in the 30 to $80 \mathrm{MHz}$ band from showers produced by primary particles with energies of about $10^{18} \mathrm{eV}$.

The systematic uncertainties of the measurement using AERA are presented. The calibration of the antenna is identified as the dominant uncertainty. The antenna response was measured in a recent calibration campaign using a flying drone. To this end, an octocopter was used to place a calibrated source at any position above the array. The campaign results in an antenna calibration accuracy of about $10 \%$.
\end{abstract}

35th International Cosmic Ray Conference - ICRC2017

10-20 July, 2017

Bexco, Busan, Korea

\footnotetext{
* Speaker.
} 


\section{Introduction}

Ultrahigh-energy cosmic rays (UHECRs) interact with the Earth's atmosphere and produce large numbers of secondary particles. Well-established detection techniques are stochastic measurements of the remaining secondary particles at ground level and direct detection of fluorescence light emitted from air molecules excited by the particle cascade. Both techniques provide information about the primary cosmic ray, e.g., the cosmic-ray energy. The primary cosmic-ray energy is an important observable for most analyses, as is the systematic uncertainty of the energy measurement. At the Pierre Auger Observatory [1] the fluorescence technique is used for the absolute energy calibration. 27 telescopes are used to reconstruct the primary cosmic-ray energy with a systematic uncertainty at the absolute scale of $14.3 \%$ [2]. Such measurements depend sensitively on atmospheric conditions, which requires extensive atmospheric monitoring efforts [1].

In recent years, measurement of radio emission from air showers in the megahertz $(\mathrm{MHz})$ regime has become a complementary detection technique $[5,6]$. The radio technique combines the reconstruction of the longitudinal and lateral shower profiles with a duty cycle close to $100 \%$ which allows for precise and accurate measurements, e.g., of the cosmic-ray energy. In the following, a new method to determine the cosmic-ray energy scale using the AERA detector $[3,4]$ is presented and its systematic uncertainties are discussed $[7,8,9]$.

\section{Radio Emission from Extensive Air Showers}

The radio emission comes from accelerated charges in the Earth's atmosphere. Two mechanisms contribute to coherent radio emission from air showers, namely the geomagnetic effect and the time-varying negative charge excess in the shower front. The geomagnetic emission is induced by acceleration of charged particles in the Earth's magnetic field forming a signal polarized according to the Lorentz force [10]. The charge excess is due to the knock-out of electrons from air molecules and annihilation of positrons in the shower front forming a radially polarized signal [11]. The radio emission from air showers can be calculated from first principles using classical electrodynamics $[12,13,14]$. The emission originates purely from the well-understood electromagnetic part of the air shower. The contribution of muons deflected in the Earth's magnetic field is negligible due to their large mass. Thus, the theoretical aspect of radio measurements is on solid grounds [5].

\section{The Auger Engineering Radio Array}

The Auger Engineering Radio Array is located within the northwest corner of the Pierre Auger Observatory. More than 150 autonomous radio stations, deployed on a regular grid with different spacings, cover about $17 \mathrm{~km}^{2}$ and are used to record radio emission in the 30 to $80 \mathrm{MHz}$ band from showers produced by primary particles with energies of over $10^{17} \mathrm{eV}$. Each radio station consists of two perpendicularly oriented antennas which are aligned to magnetic north and east with a precision better than $1^{\circ}$. The read-out system of the radio station is located in an electronics box underneath the radio antenna. Together with a GPS antenna, a solar panel and a battery, the stations are autonomously operated $24 \mathrm{~h}$ each day. Two different kinds of antenna types are used at 


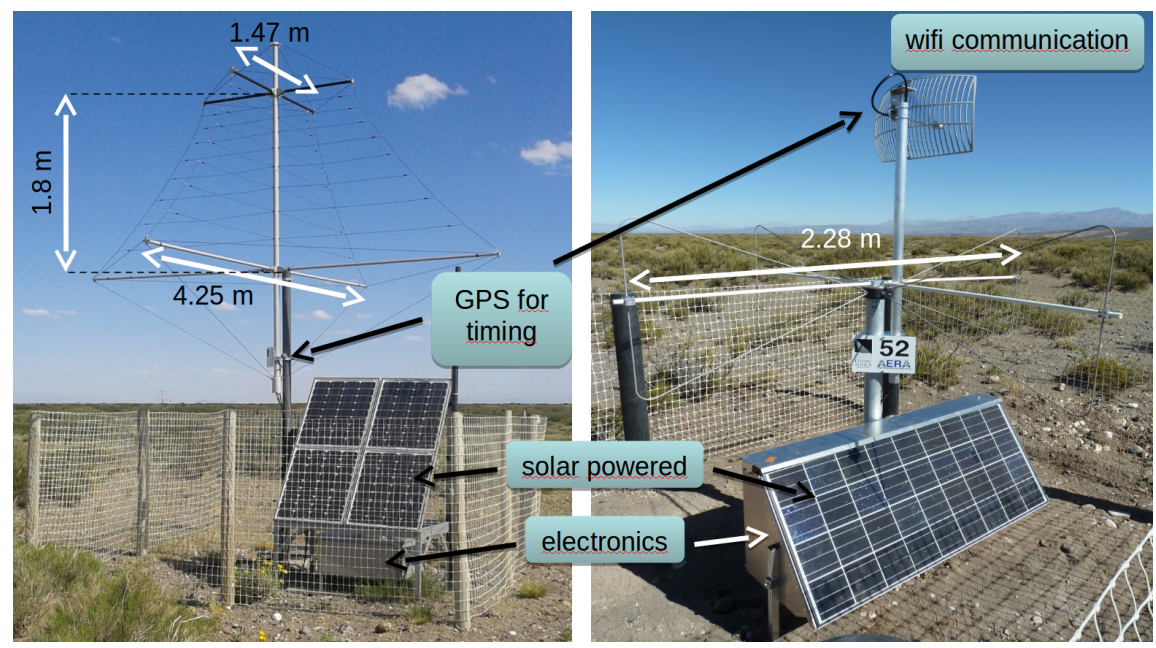

Figure 1: Photos of the LPDA (left) and butterfly (right) radio stations.

AERA: the log-periodic dipole antenna (LPDA) and the butterfly antenna [15]. Both station types are shown in Fig. 1. The following analysis is concentrated on the inner 24 radio stations with the smallest grid spacing of $144 \mathrm{~m}$. These radio stations are equipped with LPDAs.

\section{Calibration of the LPDA Radio Station}

The relation between voltage $U$ measured with each radio antenna and the incoming electric field $\vec{E}$ is described by the vector effective length (VEL). The VEL is a complex quantity for the antenna sensitivity and group delay for different incoming directions and frequencies. In Fourier space the following relation holds:

$$
U(\theta, \phi, f)=\vec{H}(\theta, \phi, f) \cdot \vec{E}(\theta, \phi, f)
$$

where $\vec{H}$ denotes the VEL depending on the arrival direction, described by spherical coordinates $\theta$ and $\phi$, and the frequency $f$. The VEL $\vec{H}$ is oriented in the plane perpendicular to the arrival direction of the signal and can be expressed as a superposition of a horizontal component $H_{\phi}$ and a component $H_{\theta}$ oriented perpendicular to $H_{\phi}$ which is called meridional component:

$$
\vec{H}=H_{\phi} \vec{e}_{\phi}+H_{\theta} \vec{e}_{\theta}
$$

The VEL of the LPDA is determined by transmitting a defined signal from a calibrated signal source from different arrival directions and measuring the LPDA response. A signal with power $P_{t}$ from a signal generator is injected to a calibrated transmitting antenna with known antenna characteristics $G_{t}$. Both are mounted underneath a GPS-controlled flying drone, a so-called octocopter. The signal $P_{r}$ received at the LPDA is measured using a spectrum analyzer. To be unaffected by near-field effects, the calibration has to be done in the far-field region which is fulfilled to a reasonable approximation at a distance of $R>2 \lambda=20 \mathrm{~m}$ for the LPDA frequency range of $30 \mathrm{MHz}$ to $80 \mathrm{MHz}$. The full calibration setup is shown on the left side of Fig. 2. 

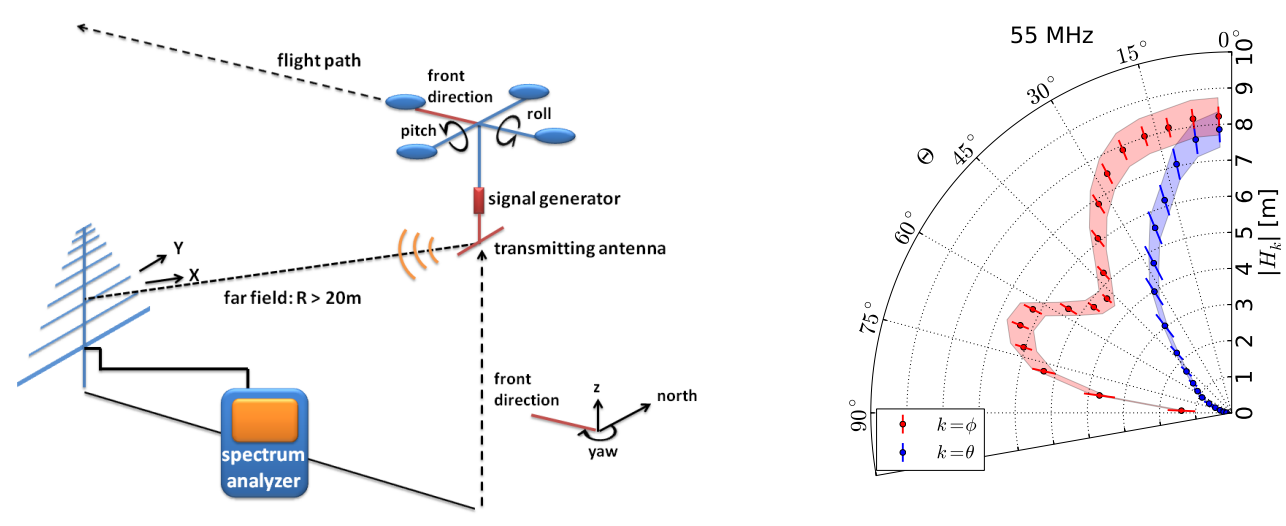

Figure 2: (left) LPDA calibration setup using an octocopter. The orientation of the octocopter is described by the yaw (twist of front measured from north in the mathematically negative direction) and the tilt by the pitch and the roll angles. [16] (right) Magnitude of horizontal and meridional LPDA VEL magnitudes (dots) and the flight-dependent uncertainties (error bars) as a function of the zenith angle in $5^{\circ}$ bins at $55 \mathrm{MHz}$. The colored bands describe the corresponding constant systematic uncertainties. [16]

The VEL magnitude is then expressed by:

$$
\left|H_{k}(\Phi, \Theta, f)\right|=\sqrt{\frac{4 \pi Z_{R}}{Z_{0}}} R \sqrt{\frac{P_{r, k}(\Phi, \Theta, f)}{P_{t}(f) G_{t}(f)}} .
$$

Here, $f$ is the signal frequency, $Z_{R}=50 \Omega$ is the readout impedance, $Z_{0} \approx 120 \pi \Omega$ is the impedance of free space, the index $k=\phi$ or $\theta$ indicates the polarization depending on the orientation of the transmitting antenna [16], and $\Phi$ and $\Theta$ denote the azimuth and zenith angle of the arrival direction. On the right side of Fig. 2 the measured horizontal and meridional VEL magnitudes of the LPDA at $55 \mathrm{MHz}$ are shown. The median overall uncertainty including statistical and systematic uncertainties is $7.4 \%$ for $\left|H_{\phi}\right|$ and $10.3 \%$ for $\left|H_{\theta}\right|$, respectively [16]. The uncertainty of the VEL magnitudes has a direct impact on the uncertainty of the cosmic-ray energy reconstruction. The energy deposit per area of the cosmic-ray radio pulse is called energy fluence. On the left side of Fig. 3 the systematic uncertainty of the square root of the energy fluence is shown which arises by propagating the uncertainties from the calibration to the electric-field reconstruction. On the right side the uncertainty is histogrammed. For zenith angles smaller than $60^{\circ}\left(80^{\circ}\right)$, the systematic uncertainty of the square root of the energy fluence is $8.8_{-1.3}^{+2.1} \%\left(9.6_{-1.9}^{+5.3} \%\right)$ in the median [16]. The square root of the energy fluence is shown because the energy fluence scales quadratically with the electric-field amplitude and the cosmic-ray energy (refer to the next section). Hence, the uncertainty of the square root of the energy fluence is the relevant uncertainty in most analyses.

\section{Reconstruction of the Cosmic-Ray Energy}

The cosmic-ray energy is reconstructed in the following way: from the measured voltage traces the incoming electric field is reconstructed using the antenna VEL. The energy fluence is calculated 

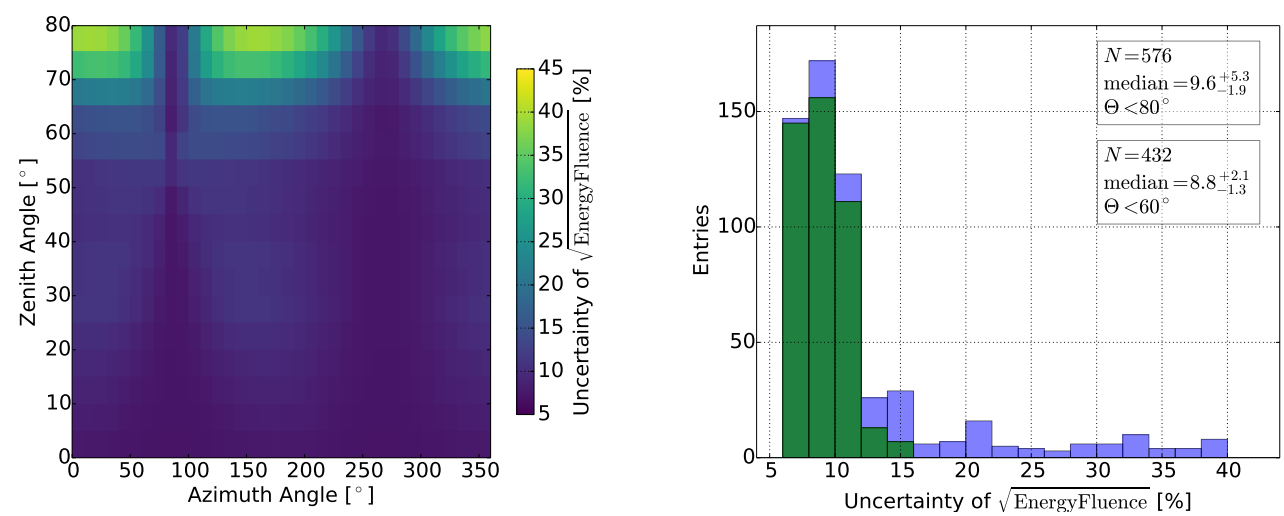

Figure 3: (left) Systematic uncertainty of the square root of the energy fluence for all arrival directions taking into account a signal polarization due to the dominant geomagnetic emission process. (right) Histogram of the systematic uncertainty of the square root of the energy fuence of signals with zenith angles smaller than $80^{\circ}$ (blue) and of signals with zenith angles smaller than $60^{\circ}$ (green). [16]

by a time integral of the Poynting vector of the reconstructed electric field in each station. A two-dimensional lateral distribution function (LDF) [17] is fitted, taking into account asymmetries resulting from the two emission processes. The LDF shape is best understood within the shower plane with one axis perpendicular to the shower direction $\vec{v}$ and the Earth's magnetic field $\vec{B}: \vec{v} \times \vec{B}$, and the perpendicular axis: $\vec{v} \times(\vec{v} \times \vec{B})$. On the left side of Fig. 4, the energy fluence distribution of a measured cosmic ray and the corresponding LDF fit are presented. The radiation energy, which is the total amount of energy transferred from the primary cosmic ray into radio emission, is then obtained by integrating the LDF fit over the area. Then, the radiation energy is corrected for the geometric dependence of the geomagnetic emission by dividing by $\sin ^{2}(\alpha)$ where $\alpha$ is the angle between $\vec{v}$ and $\vec{B}$. On the right side of Fig. 4, the corrected radiation energies of 126 air showers are shown, measured with the LPDA radio stations at AERA and cross-calibrated with the well-understood data of the Pierre Auger Observatory. The observed radiation energy scales quadratically with the cosmic-ray energy. From the scatter around the calibration curve, the cosmicray energy resolution of AERA is determined to be $22 \%$, improving to $17 \%$ for events with at least five stations with signal, which is of the same order of magnitude as the energy resolution of the Auger surface detector [2].

\section{Independent Energy Scale Using AERA}

In the previous section the measured radiation energy is calibrated using the information of the baseline detectors of the Pierre Auger Observatory. Instead, the energy calibration can be done by using the theoretical prediction of the radiation energy. The idea is illustrated on the left side of Fig. 5. The procedure to determine the radiation energy is divided into an experimental part, as explained in the previous section, and a theoretical part. The radio emission comes from accelerated charges in the Earth's atmosphere and originates from the well-understood electromagnetic part of the air shower. As soon as the radio emission stops at the end of the air-shower development, the 

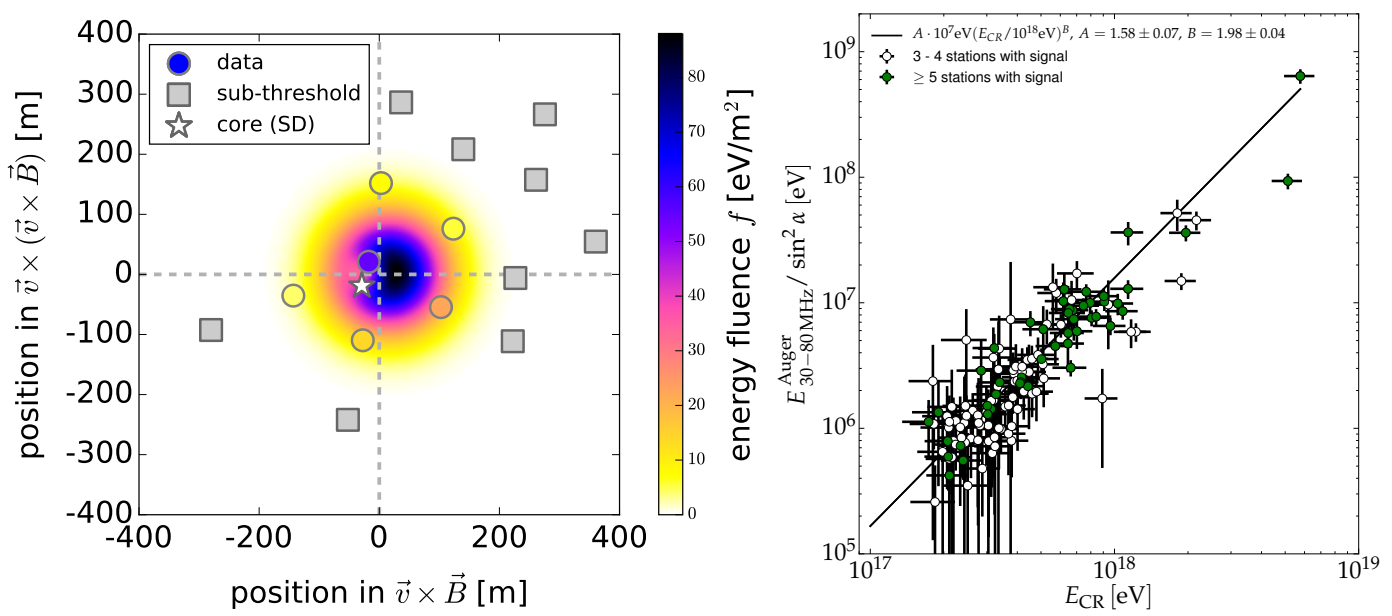

Figure 4: (left) Energy fluence for an extensive air shower with an energy of $4.4 \times 10^{17} \mathrm{eV}$ and a zenith angle of $25^{\circ}$ measured with AERA (colored circles). The center indicates the shower core reconstructed with the radio data. The colored background indicates the two-dimensional LDF fit. The white star marks the shower core reconstructed using the surface detector data of the Pierre Auger Observatory. [7, 8] (right) The corrected radiation energy in relation to the cosmic-ray energy measured with the baseline detectors of the Pierre Auger Observatory. Events with 3 and 4 stations with signal are marked as white circles and in case of more than 5 stations with signal are marked as green circles. [7, 8]

radiation energy stays constant because the Earth's atmosphere is essentially transparent for radio waves in the VHF band. Thus, measurement and theoretical prediction of the radiation energy are directly comparable. For a detailed discussion about the theoretical part refer to [14]. In the following, the CoREAS simulation code [13] is used to simulate air showers and their radio emission. CoREAS is a microscopic simulation code, where the radiation of each single particle is calculated by first principles of classical electrodynamics, and then superposed to the full radio emission. As the emission originates from the electromagnetic part of the air shower, the radiation energy correlates best with the energy of the electromagnetic cascade. In addition to the geometric dependence of the geomagnetic emission process, a second dependence on the air density at the shower maximum was identified and parametrized. This corrected radiation energy is presented as a function of the energy in the electromagnetic cascade on the right side of Fig. 5. A quadratic relation with a scatter of less than $3 \%$ is observed [14]. To obtain the cosmic-ray energy from the electromagnetic shower energy, the invisible energy needs to be taken into account, e.g., neutrinos and high-energy muons do not contribute to the radiation energy. This can be done using a parametrization that was obtained from measurements [18].

In the following, the currently estimated systematic uncertainties with respect to the cosmic-ray energy are briefly discussed [9]. The uncertainty from the antenna calibration of the radio detector is identified as the dominant uncertainty of the cosmic-ray energy measurement with AERA.

\section{Experimental uncertainties}

The experimental uncertainties involve the systematic uncertainties of the radio detector, the ana$\log$ signal chain from the radio antenna to the digitizer and the fitted LDF model. With a recent 

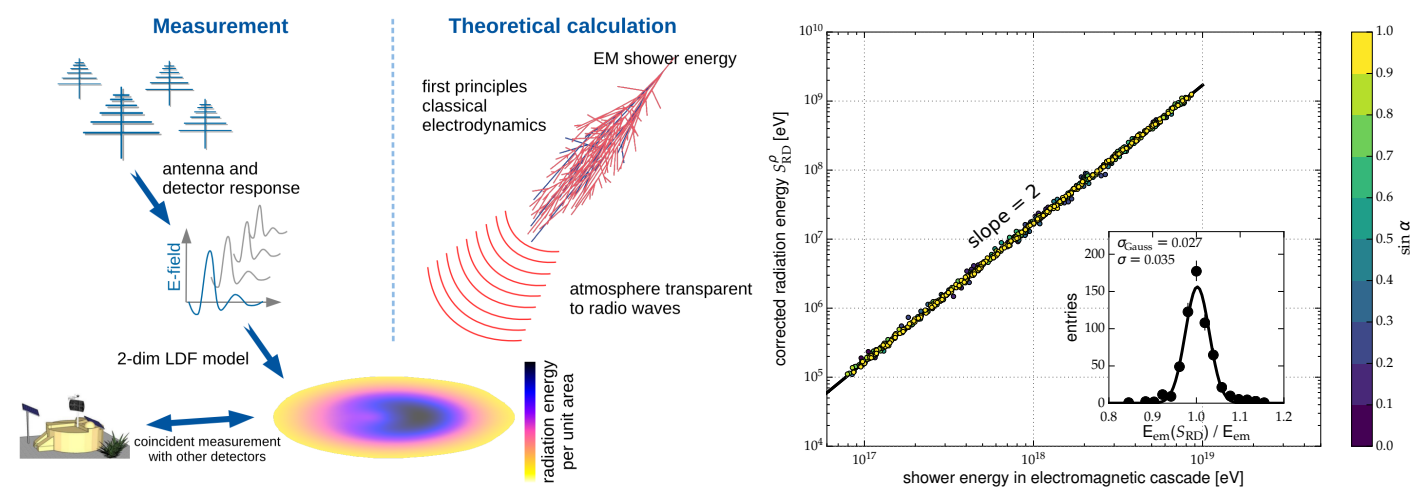

Figure 5: (left) Idea of the determination of the energy scale from first-principles calculations using radio measurements. [9] (right) The corrected radiation energy estimator vs. the energy in the electromagnetic cascade from CoREAS simulations. Adapted from [14].

calibration campaign the systematic uncertainty was lowered to about $10 \%$ as described in section 4. For more details refer to [16]. The analog signal chain is measured with a systematic uncertainty below $1 \%$. The systematic uncertainty of the LDF model contributes about $2.5 \%$ [8].

\section{Uncertainties of the relation between radiation energy and electromagnetic shower energy}

The calculation of the radio emission is purely based on classical electrodynamics. Therefore, it has no free parameters and thus no systematic uncertainty. Nevertheless, the modeling of the electromagnetic air-shower component and approximations made in the simulation code result in further uncertainties. The influence of all known approximations have been checked and are small compared to the experimental uncertainties [9]. Additional theoretical uncertainties cannot be excluded but the form of the measured radio pulse and the measured signal distribution on ground are in good agreement with the CoREAS simulation, e.g., refer to [19]. Thus, uncertainties of the relation between radiation energy and electromagnetic shower energy are expected to be small compared to the experimental uncertainties.

\section{Environmental uncertainties}

Changing atmospheric conditions, i.e., changing density profiles and varying refractivity, result in a scatter of about $1 \%$ during the course of the year which is taken as estimated uncertainty [9]. Furthermore, changing ground conditions, e.g., ground permittivity and ground conductivity, change the reflectivity of the ground and thereby directly impact the radio detector directional sensitivity. An LPDA simulation study reveals an average change of the antenna response pattern of $1 \%$ for different realistic ground conditions at the AERA site [16].

\section{Invisible energy correction}

The systematic uncertainty of the parametrization to obtain the cosmic-ray energy from the electromagnetic shower energy was determined to be $3 \%$ at $10^{18} \mathrm{eV} \mathrm{[18].}$

\section{Conclusion}

The Auger Engineering Radio Array is the largest cosmic-ray radio detector worldwide and 
enables the measurement of the radio emission of extensive air showers. This provides information, e.g., about the cosmic-ray energy. Well-calibrated radio stations enable both precise and accurate measurements of the radio signal. In a recent calibration campaign, the magnitude of the horizontal and meridional VEL of the LPDA radio stations has been measured. The systematic uncertainty of the LPDA VEL propagates to a systematic uncertainty of $8.8 \%$ of the square root of the energy fluence. The radiation energies of 126 air showers have been measured and compared with the cosmic-ray energies measured with the baseline detectors of the Pierre Auger Observatory. A quadratic relation between radiation energy and cosmic-ray energy is found. AERA obtains a cosmic-ray energy resolution of $17 \%$ for events with more than 5 radio stations with signal.

Furthermore, a new method to independently determine the cosmic-ray energy scale using radio is presented combining radio measurements of the radiation energy with theoretical calculations from first principles using classical electrodynamics. The systematic uncertainties of the cosmic-ray energy scale using AERA are briefly discussed. The uncertainty due to the antenna calibration is identified as the dominant one. Finally, the systematic uncertainty of the cosmic-ray energy scale using AERA is expected to be at the same level as the systematic uncertainty of the cosmic-ray energy scale using the fluorescence detector of the Pierre Auger Observatory.

\section{References}

[1] A. Aab et al. (Pierre Auger Collaboration), Nucl. Instrum. Meth. A 798 (2015) 172-213

[2] V. Verzi for the Pierre Auger Collaboration, Proc. 33rd ICRC, Rio de Janeiro, Brazil (2013) 928

[3] J. Schulz for the Pierre Auger Collaboration, Proc. 34th ICRC, The Hague, The Netherlands (2015) PoS(ICRC2015)615

[4] E. Holt for the Pierre Auger Collaboration, Proc. 35th ICRC, Busan, South Korea (2017)

[5] T. Huege, Phys. Rep. 620 (2016) 1-52

[6] F.G. Schröder, Prog. in Part. and Nucl. Phys. 93 (2017) 1-68

[7] A. Aab et al. (Pierre Auger Collaboration), Phys. Rev. Lett. 116 (2016) 241101

[8] A. Aab et al. (Pierre Auger Collaboration), Phys. Rev. D 93 (2016) 122005

[9] C. Glaser, PhD thesis, RWTH Aachen University (2017)

[10] F.D. Kahn, I. Lerche, Proc. R. Soc. Lond. A 289 (1966) 206-213

[11] G. Askaryan, Soviet Phys. JETP Lett. (USSR) 14 (1962) 441

[12] J. Alvarez-Muñiz, W.R. Carvalho and E. Zas, Astropart. Phys. 35 (2012) 325-341

[13] T. Huege, M. Ludwig and C.W. James, Proc. AIP Conf. 1535 (2013) 128-132

[14] C. Glaser, M. Erdmann, J.R. Hörandel, T. Huege and J. Schulz, JCAP 09 (2016) 024

[15] P. Abreu et al (Pierre Auger Collaboration), J. Instrum. 7 (2012) P10011

[16] A. Aab et al (Pierre Auger Collaboration), submitted to J. Instrum. (2017)

[17] A. Nelles et al., Astropart. Phys 60 (2015) 13-24

[18] M.J. Tueros for the Pierre Auger Collaboration, Proc. 33rd ICRC, Rio de Janeiro, Brazil (2013) 705

[19] S. Buitink et al., Nature 531 (2016) 70-73 